\title{
Pleurectomía parietal y decorticación pulmonar por cirugía torácica asistida por video (VATS) monopuerto versus toracotomía
}

\author{
Parietal pleurectomy and pulmonary decortication by single-port \\ video-assisted thoracic surgery (VATS) versus thoracotomy
}

\author{
Anuar Alonso Sáez-Martínez $\mathbb{D}$, Daniel José Jaller-Salleg² $\mathbb{D}$, Enrique Carlos Ramos-Clason³ $\mathbb{D}$, \\ Paula Andrea Usta-Tirado ${ }^{4}$ D
}

1 Médico, especialista en Cirugía general, Cartagena, Colombia.

2 Médico, especialista en Cirugía general y Cirugía de Tórax; docente, postgrado Cirugía General, Universidad del Sinú EBZ, Seccional Cartagena, Colombia.

3 Médico, magíster en Salud pública; director de investigaciones, postgrados Médico-Quirúrgicos, Universidad del Sinú EBZ, Seccional Cartagena, Colombia.

4 Médico, Universidad del Norte, Barranquilla, Colombia.

\section{Resumen}

Introducción. El avance de la cirugía torácica abierta a cirugía torácica asistida por vídeo por tres puertos, y sus posteriores efectos en la recuperación de los pacientes, conllevó al desarrollo de la técnica por un solo puerto, que ha mostrado beneficios en el postoperatorio.

El objetivo de este estudio fue comparar los resultados postquirúrgicos de los pacientes sometidos a pleurectomía parietal y decorticación pulmonar toracoscópica asistida por video monopuerto y los obtenidos por toracotomía convencional, en una clínica de cuarto nivel, entre 2016 y 2019.

Métodos. Estudio descriptivo, en el que se incluyeron 79 pacientes llevados a pleurectomía parietal y decorticación pulmonar por toracoscopia asistida por vídeo monopuerto y 25 pacientes operados por toracotomía convencional. Se evaluaron variables sociodemográficas, clínicas y postoperatorias. Se utilizaron las pruebas de $\mathrm{Chi}^{2}$ o de Fisher y las pruebas t de Student y Mann Whitney.

Resultados. La mediana de edad fue menor en el grupo de pacientes operados por toracotomía convencional (28 años, RIC: 26-48, p=0,0005). No hubo diferencia en los tiempos quirúrgicos. Se encontró menor intensidad del dolor y disminución en los días con tubo de tórax, uso de antibióticos, días de UCI y días de estancia hospitalaria en el grupo de pacientes operados por toracoscopia asistida por vídeo monopuerto $(\mathrm{p}<0,05)$.

Fecha de recibido: 11/08/2020 - Fecha de aceptación: 14/10/2020 - Fecha de publicación en línea: 12/02/2021

Correspondencia: Anuar Alonso Sáez-Martínez, Carrera 3 \# 63-24, Apartamento 201, Montería, Colombia.

Teléfono: 57 (4) 7569441. Correo electrónico: anuarsaez11@gmail.com

Citar como: Sáez-Martínez AA, Jaller-Salleg DJ, Ramos-Clason EC, Usta-Tirado PA. Pleurectomía parietal y decorticación pulmonar por cirugía torácica asistida por video (VATS) monopuerto versus toracotomía. Rev Colomb Cir. 2021;36:275-82. https://doi.org/10.30944/20117582.746

Este es un artículo de acceso abierto bajo una Licencia Creative Commons - BY-NC-ND https://creativecommons.org/licenses/by-ncnd/4.0/deed.es 
Discusión. Este estudio refuerza la tendencia de mejores resultados postquirúrgicos, menos días de uso del tubo de tórax, uso de antibióticos, necesidad de UCI y días de estancia hospitalaria general con la técnica asistida por vídeo monopuerto comparado con la toracotomía abierta convencional.

Palabras clave: cirugía torácica; cirugía torácica asistida por video; toracotomía; lobectomía; decorticación pleural; evaluación de resultados de intervenciones terapéuticas.

\begin{abstract}
Introduction. The advancement from open to video-assisted thoracic surgery through three ports, and its subsequent effects on the recovery of patients, led to the development of the single port technique, which has shown benefits in the postoperative period. The objective of this study was to compare the postsurgical results of patients undergoing parietal pleurectomy and video-assisted single-port thoracoscopic pulmonary decortication to those obtained by conventional thoracotomy, in a fourth level clinic, between 2016 and 2019.
\end{abstract}

Methods. Descriptive study, in which 79 patients underwent parietal pleurectomy and pulmonary decortication by single-port video-assisted thoracoscopy and 25 patients operated by conventional thoracotomy were included. Sociodemographic, clinical and postoperative variables were evaluated. The Chi-square or Fisher tests, and the $t$ Student and Mann Whitney $t$ tests were used.

Results. The median age was lower in the conventional thoracotomy group (28 years; IQR: 26-48; $p=0.0005$ ). There were no differences in surgical times. Lower pain level, and a decreased in days with chest tube, antibiotic use, need for ICU and of hospital stay were reported in the single-port video-assisted thoracoscopy group compared to conventional thoracotomy technique $(\mathrm{p}<0.05)$.

Discussion. This study reinforces the trend of better postsurgical results, fewer days of chest tube use, use of antibiotics, need for ICU and days of general hospital stay with the single-port video-assisted technique compared to conventional open thoracotomy.

Key words: thoracic surgery; video-assisted thoracic surgery; thoracotomy; lobectomy; pleural decortication; evaluation of results of therapeutic interventions.

\section{Introducción}

La cirugía torácica asistida por video (Video-Assisted Thoracoscopic Surgery, VATS) surgió con el desarrollo del monitor de video e inicialmente utilizaba tres puertos para la introducción de los instrumentos endoscópicos ${ }^{1}$. La VATS monopuerto permite realizar el mismo procedimiento a través de una sola incisión, generalmente ubicada a lo largo de la línea axilar.

La evolución de los procedimientos quirúrgicos va dirigida a disminuir la magnitud de los abordajes, garantizando resultados iguales o más eficaces que los procedimientos convencionales ${ }^{2}$. En la literatura existe poca evidencia que compare ambos procedimientos, entre ellos, dos ensayos aleatorios controlados de
VATS versus cirugía abierta ${ }^{3,4}$, y el estudio VIOLET, fundamentado en la hipótesis de que VATS conducirá a un menor trauma tisular que la cirugía abierta $\mathrm{y}$, por lo tanto, a una mejor recuperación de varios aspectos de la calidad de vida, relacionados con la salud en el período postoperatorio temprano ${ }^{5}$.

Esto incentiva a realizar un estudio que muestre las diferencias del uso de la VATS monopuerto en comparación con la toracotomía convencional. Por consiguiente, el presente estudio busca comparar los resultados postquirúrgicos de los pacientes sometidos a pleurectomía parietal y decorticación pulmonar por VATS monopuerto y aquellos realizados por toracotomía convencional, entre 2016 y 2019. 


\section{Métodos}

Se realizó un estudio observacional descriptivo comparativo de 104 pacientes adultos que tuvieron procedimientos de decorticación pulmonar y pleurectomía parietal, por VATS monopuerto o toracotomía por técnica convencional, realizados en IMAT Oncomédica, una clínica de cuarto nivel de complejidad. Se excluyeron las pacientes embarazadas, pediátricos y con el procedimiento realizado de forma secundaria en el marco de otro procedimiento principal. El centro médico proporcionó la base de datos con el registro de todas las historias clínicas de pacientes a quienes se les realizó decorticación pulmonar y pleurectomía parietal.

La toracotomía posterolateral se realizó en aquellos pacientes con hallazgos tomográficos prequirúrgicos de proceso patológico complejo, como múltiples adherencias o amplia zona de tabiques, entre otros, que predecian mayor tiempo quirúgico y riesgo de complicaciones.

Se estudiaron variables preoperatorias sociodemográficas, como edad, sexo y tipo de afiliación al Sistema de Seguridad Social en Salud; clínicas, como índice de masa corporal (IMC), estado nutricional según el índice de masa corporal (IMC) y antecedente de tabaquismo. Además, se tuvieron en cuenta datos relacionados con el procedimiento, como la indicación (clasificada en infecciosa, oncológica o traumática), lateralidad de la lesión y ámbito de realización del procedimiento (programado o por urgencias). Dentro de las variables intraoperatorias, se consignaron datos como el tiempo quirúrgico, estimación del sangrado, necesidad de transfusión, número de tubo de tórax, necesidad de conversión a toracotomía, lobectomía, causa de lobectomía, realización, método de pleurodesis, y reexpansión pulmonar (medida subjetivamente de forma visual en una calificación de 0 a $100 \%$ ).

Las variables postoperatorias evaluadas fueron dolor inmediato al postoperatorio, necesidad y tipo de reintervención, y días con tubo de tórax. Los criterios para retiro de tubo de tórax fueron buena ventilación del campo pulmonar afectado, buena expansión pulmonar, drenaje menor a 100 $\mathrm{ml}$ en 24 horas. Se tuvieron en cuenta también, desarrollo de infección del sitio operatorio (ISO), realización de cultivo, número de días de antibióticos, necesidad de UCI y días de estancia en UCI, días de estancia hospitalaria, reingreso, muerte y causa de muerte. Los criterios de ingreso a UCI fueron inestabilidad hemodinámica, falla ventilatoria y necesidad de reintervención por complicaciones.

Todas las variables fueron tabuladas en una matriz de Microsoft Excel@ para su posterior análisis estadístico. El análisis estadístico descriptivo de variables cualitativas se realizó con el cálculo de frecuencias absolutas y relativas. Las variables cuantitativas se analizaron con medidas de tendencia central tipo promedio aritmético $(\bar{X})$ o mediana (Me), reportadas con desviación estándar (DE) y rango intercuartílico (RIC), utilizadas según la distribución de normalidad estimada por la prueba de Kolmogorov Smirnov. Para comparar entre el grupo de VATS monopuerto y toracotomía convencional, se utilizó según necesidad el Test de $\mathrm{Chi}^{2}$ o el test exacto de Fisher en variables cualitativas. La comparación de las variables cuantitativas se realizó con las pruebas t Student en variables paramétricas y la U de Mann Whitney en las no paramétricas. Para todas las pruebas estadísticas un valor de p menor de 0,05 fue considerado como estadísticamente significativo.

\section{Resultados}

En el periodo de estudio se identificaron 104 pacientes sometidos a pleurectomía parietal y decorticación pulmonar, de las cuales 79 fueron cirugía torácica asistida por video (VATS) monopuerto y las 25 restantes fueron por toracotomía posterolateral. La edad promedio fue de 49 años (RIC: $35-70$ ) en el grupo VATS y de 28 años (RIC: 26-48) en el grupo de toracotomía $(p=0,0005)$. El sexo masculino fue más frecuentemente intervenido, con $68,4 \%$ en el grupo VATS y $52 \%$ en el de toracotomía. El régimen de seguridad social en salud más frecuente fue el subsidiado, con más del $59 \%$ de los casos en ambos grupos (tabla 1).

El promedio de IMC fue de $24,87 \pm 3,1 \mathrm{~kg} / \mathrm{m}^{2}$ en el grupo de VATS y de $22,27 \pm 4,3 \mathrm{~kg} / \mathrm{m}^{2}$ en el de toracotomía $(p=0,0026)$. El estado nutricional 
Tabla 1. Características sociodemográficas, antecedentes y contexto general de la lesión estratificados por técnica utilizada.

\begin{tabular}{|c|c|c|c|}
\hline & $\begin{array}{c}\text { Videotoracoscopia } \\
\mathrm{N}=79 \\
\mathrm{n}(\%)\end{array}$ & $\begin{array}{l}\text { Toracotomía } \\
\mathrm{N}=25 \\
\mathrm{n}(\%)\end{array}$ & Valor $p$ \\
\hline Edad, años; Me (RIC) & $49(35-70)$ & $28(26-48)$ & 0,0005 \\
\hline \multicolumn{4}{|l|}{ Sexo } \\
\hline Masculino & $54(68,4)$ & $13(52,0)$ & \multirow[t]{2}{*}{0,1365} \\
\hline Femenino & $25(31,6)$ & $12(48,0)$ & \\
\hline \multicolumn{4}{|c|}{ Régimen de Seguridad Social en Salud } \\
\hline Subsidiado & $47(59,5)$ & $16(64,0)$ & 0,6877 \\
\hline Contributivo & $27(34,2)$ & $9(36,0)$ & 0,8663 \\
\hline Especial & $4(5,1)$ & $0(0,0)$ & 0,5700 \\
\hline \multirow{3}{*}{$\begin{array}{l}\text { Particular } \\
\text { Índice de masa corporal, } \mathrm{kg} / \mathrm{m}^{2} \\
\text { media } \pm \mathrm{DE} \\
\text { Bajo peso }\end{array}$} & $1(1,3)$ & $0(0,0)$ & 0,5737 \\
\hline & $24,87 \pm 3,1$ & $22,27 \pm 4,3$ & 0,0026 \\
\hline & $2(2,5)$ & $3(12,0)$ & 0,0885 \\
\hline Normal & $40(50,6)$ & $12(48,0)$ & 0,8185 \\
\hline Sobrepeso & $32(40,5)$ & $5(20,0)$ & 0,0619 \\
\hline Obesidad & $3(3,8)$ & $0(0,0)$ & 0,3228 \\
\hline Sin dato & $0(0,0)$ & $5(20,0)$ & -- \\
\hline \multicolumn{4}{|l|}{ Antecedentes personales } \\
\hline Tabaquismo & $46(58,2)$ & $10(40,0)$ & 0,1110 \\
\hline \multicolumn{4}{|l|}{ Indicación de la cirugía } \\
\hline Infecciosa & $26(32,9)$ & $11(44,0)$ & 0,3128 \\
\hline Oncológica & $19(24,1)$ & $7(28,0)$ & 0,6910 \\
\hline Traumática & $14(17,7)$ & $3(12,0)$ & 0,7569 \\
\hline Inespecífica & $20(25,3)$ & $4(16,0)$ & 0,4216 \\
\hline \multicolumn{4}{|l|}{ Lateralidad } \\
\hline Derecha & $57(72,2)$ & $14(56,0)$ & 0,1304 \\
\hline Izquierda & $22(26,6)$ & $10(40,0)$ & 0,2512 \\
\hline Bilateral & $1(1,3)$ & $1(4,0)$ & 0,4247 \\
\hline \multicolumn{4}{|l|}{ Ámbito } \\
\hline Urgencias & $57(72,2)$ & $16(67,0)$ & 0,4373 \\
\hline Electiva & $22(27,8)$ & $9(36,0)$ & \\
\hline
\end{tabular}

* Me: media; RIC: rango intercuartílico; DE: desviación estándar.

más frecuente en ambos grupos fue el normal, seguido del sobrepeso. El antecedente de tabaquismo se observó en el 58,2 \% de los pacientes del grupo VATS y $40 \%$ del grupo de toracotomía. La indicación más frecuente de los procedimientos fue infecciosa en ambos grupos. No existieron diferencias estadísticamente significativas entre los grupos al comparar estado nutricional, indicación, lateralidad ni ámbito de realización del procedimiento.
La mediana del tiempo quirúrgico fue de 70 minutos (RIC: 60-90) en el grupo VATS y de 75 min (RIC: 60-90) en el grupo de toracotomía (tabla 2). El sangrado fue estimado en menos de 500 ml en el 83,5 \% de VATS y $76 \%$ de toracotomía y hubo necesidad de transfusión en menos del $9 \%$ en ambos grupos. La necesidad de conversión a técnica abierta en el grupo VATS fue de 2,5\%. Se practicó lobectomía en el 11,4 \% de los pacientes del grupo VATS y en el 20 \% del grupo de toraco- 
Tabla 2. Datos intraoperatorios y postoperatorios por técnica quirúrgica utilizada.

\begin{tabular}{|c|c|c|c|}
\hline & $\begin{array}{l}\begin{array}{l}\text { Videotoracoscopia } \\
\mathrm{N}=79 \\
\mathrm{n}(\%)\end{array} \\
\end{array}$ & $\begin{array}{l}\text { Toracotomía } \\
\mathrm{N}=25 \\
\mathrm{n}(\%)\end{array}$ & Valor $p$ \\
\hline Tiempo quirúrgico, minutos; Me (RIC) & $70(60-90)$ & $75(60-90)$ & 0,7524 \\
\hline \multicolumn{4}{|l|}{ Sangrado } \\
\hline Menos de $500 \mathrm{ml}$ & $66(83,5)$ & $19(76,0)$ & 0,3948 \\
\hline 500 ml o más & $13(16,5)$ & $6(24,0)$ & \\
\hline \multicolumn{4}{|l|}{ Transfusión } \\
\hline Glóbulos rojos, unidades & $4(5,1)$ & $2(8,0)$ & 0,5830 \\
\hline Tamaño del Tubo de tórax; Me (RIC) & $30(28-30) \mathrm{Fr}$ & $30(20-31) \mathrm{Fr}$ & 0,1664 \\
\hline Porcentaje de reexpansión; Me (RIC) & $90(80-100)$ & $90(80-100)$ & 0,6168 \\
\hline Conversión & $1(1,3)$ & $0(0,0)$ & 0,5737 \\
\hline Lobectomía & $9(11,4)$ & $5(20,0)$ & 0,3162 \\
\hline Nódulo & $3(3,8)$ & $1(4,0)$ & 0,9635 \\
\hline Absceso & $3(3,8)$ & $0(0,0)$ & 0,3251 \\
\hline Bula & $1(1,3)$ & $0(0,0)$ & 0,5737 \\
\hline \multirow{3}{*}{$\begin{array}{l}\text { Neumonectomía total } \\
\text { Patrón sospechoso micro nodular } \\
\text { del lóbulo inferior } \\
\text { Cierre de fístula broncopleural }\end{array}$} & $1(1,3)$ & $0(0,0)$ & 0,5737 \\
\hline & $1(1,3)$ & $0(0,0)$ & 0,5737 \\
\hline & $0(0,0)$ & $1(4,0)$ & 0,2403 \\
\hline Destrucción del parénquima & $0(0,0)$ & $1(4,0)$ & 0,2403 \\
\hline Lesión pulmonar basal congénita & $0(0,0)$ & $1(4,0)$ & 0,2403 \\
\hline Metástasis & $0(0,0)$ & $1(4,0)$ & 0,2403 \\
\hline Pleurodesis & $5(6,3)$ & $0(0,0)$ & 0,3335 \\
\hline Yodo & $4(5,1)$ & $0(0,0)$ & 0,5700 \\
\hline Talco & $1(1,3)$ & $0(0,0)$ & 0,5737 \\
\hline
\end{tabular}

* Me: media; RIC: rango intercuartílico; DE: desviación estándar.

tomía, mientras la pleurodesis fue realizada en el 6,3 \% de los casos del grupo VATS, pero no se realizó en el grupo de técnica abierta.

Al comparar la evolución postoperatoria de los pacientes por técnicas quirúrgicas, se observó mediana de 5 (RIC: 4-7) para la intensidad del dolor en el grupo de VATS monopuerto y de 8 (RIC: 5 -8) en el grupo de toracotomía $(p=0,0001)$. Los pacientes describieron la intensidad del dolor como severa en el $52 \%$ del grupo de toracotomía y sólo en el 8,9 \% del grupo de VATS ( $\mathrm{p}<0,0001)$. La necesidad de reintervención fue del 10,1\% en el grupo de VATS y del $16 \%$ en el de toracotomía. Las causas de reintervención más frecuentes fueron el hemotórax coagulado y el empiema en ambos grupos.

La mediana de tiempo con el tubo de tórax fue de 4 días (RIC: 2-6) en el grupo de VATS y de 7 días (RIC: 4-15) en el grupo de toracotomía $(\mathrm{p}=0,0031)$. La ISO se presentó en menos del 1,5 $\%$ de los dos grupos y la mediana de tiempo con antibióticos fue de 7 días (tabla 3). La necesidad de UCI fue del 26,6\% en el grupo de VATS y del 68 $\%$ en el de toracotomía ( $\mathrm{p}=0,0001)$. La mediana de estancia en UCI fue de 8 días (RIC: 3-24) en el grupo de VATS y de 14 días (RIC: 9-20) en el grupo de toracotomía $(\mathrm{p}=0,1153)$ y la mediana de estancia hospitalaria fue de 10 días (RIC: 7-15) en el grupo de VATS y de 15 días (RIC: 9-29) en el grupo de toracotomía $(p=0,0282)$. No hubo reingresos en el grupo de VATS mientras fue del $4 \%$ en grupo de toracotomía $(p=0,5737)$. Finalmente, fallecieron el 6,3 \% de los pacientes del grupo de VATS y el $12 \%$ del grupo de toracotomía ( $p=0,3949$ ); la principal causa de muerte en el grupo de VATS fue paro cardiaco $(5,1 \%)$ y en el grupo de toracotomía fue falla multiorgánica (8\%).

\section{Discusión}

La toracotomía ha sido utilizada tradicionalmente, como una técnica segura, para la decorticación pulmonar, especialmente en empiemas estadios 
II y III, pero recientemente se ha demostrado la eficacia de la VATS en el tratamiento del empiema pleural ${ }^{6}$.

En la búsqueda de minimizar el trauma quirúrgico, la VATS monopuerto es el procedimiento más novedoso, que limita el trauma quirúrgico a un solo espacio intercostal, con lo que reduce el dolor postoperatorio y mejora la recuperación. Se ha publicado poco sobre el uso de VATS monopuerto en decorticación pulmonar y pleurectomía, a pesar de que cada vez se ha adaptado más para el manejo de derrames pleurales recurrentes no complicados, estadificación preoperatoria en cáncer de pulmón, tratamiento de neumotórax espontáneo, e incluso para resecciones pulmonares anatómicas complejas ${ }^{7}$. Un número creciente de centros ha informado de su experiencia con VATS monopuerto en desbridamiento y decorticación para empiema, junto con otros procedimientos menores, como biopsias pleurales y resecciones en cuña, que representan la mayor parte de su serie de $\operatorname{casos}^{8}$.

En la revisión bibliográfica no se encontraron publicaciones que compararan directamente el uso de VATS monopuerto versus toracotomía en procedimientos como decorticación y pleurectomía parietal. En el presente estudio se observó que los procedimientos por VATS monopuerto se realizaron en pacientes con un rango de edades que osciló entre la cuarta y séptima década de la vida, que son pacientes con más comorbilidades y mayor riesgo de complicaciones. Estos hallazgos son similares a lo encontrado por Onaitis en 2006, con un promedio de edad de 65 años ${ }^{9}$.

Los pacientes sometidos a VATS experimentaron un dolor leve o moderado, mientras que los sometidos a toracotomía experimentaron dolor moderado a severo, según la escala del dolor. Estos hallazgos coinciden con los presentados por Ismail et. al., donde el nivel de dolor postoperatorio resultó ser muy bajo (valor medio 1,83 $\pm 1,53$ ) según la escala visual análoga del dolor EVA ${ }^{6}$. Adicionalmente, la duración media del dolor fue de 2,63 $\pm 2,23$ días, con una resolución casi total después de la extracción del tubo de toráx (valor medio $0,33 \pm 0,92$ ) en los pacientes a quienes se les realizó decorticación por VATS monopuerto ${ }^{6}$.

La duración del tubo de tórax fue menor en pacientes sometidos a VATS monopuerto que los sometidos a toracotomía posterolateral. Estos datos son consistentes con los resultados de Pan et. al., en un metaanálisis comparando decorticación pulmonar mediante técnica VATS versus toracotomía. Se seleccionaron 5 artículos que cumplían con criterios de inclusión encontrando menor el tiempo de tubo de tórax en el grupo VATS que en el grupo toracotomía, con una diferencia de medias de $-1,52\left(\mathrm{IC}_{95 \%}-2,55 \mathrm{a}-0,48 ; \mathrm{p}=0,004\right)^{10}$.

Se encontró sesgo de información en la variable sangrado intraoperatorio, ya que su medición no se realizó de forma específica, sino que se aproximó y categorizó en mayor o menor a $500 \mathrm{ml}$, por lo cual se denota un volumen medio de la pérdida de sangre intraoperatoria similar en los dos grupos. En el estudio de Chan et. al., donde compararon VATS versus toracotomía en pacientes con indicación de decorticación, no se encontró diferencia estadísticamente significativa ${ }^{11}$. Se esperaba una menor tasa de sangrado por la técnica VATS monopuerto, sin embargo, el doble de pacientes requirió transfusión sanguínea.

El tiempo quirúrgico, porcentaje de reexpansión pulmonar, reingreso, días de estancia en UCI y muertes fueron similares en los dos grupos. Los días de tratamiento antibiótico y los días de hospitalización fueron menores en el grupo sometido a VATS monopuerto comparado con toracotomía posterolateral, con diferencia estadísticamente significativa, similar a lo descrito por Cardillo et. al. ${ }^{12}$, donde la estancia hospitalaria en pacientes sometidos a decorticacion por técnica VATS fue menor comparado con la toracotomía posterolateral (6,8 versus 10,4 días) mientras, van Middendorp et. al. ${ }^{7}$ reportaron 18 días de estancia hospitalaria en su estudio retrospectivo. Esta amplia variación se puede deber a que los pacientes eran dados de alta por el servicio de cirugía para egreso con rehabilitación pulmonar ambulatoria, a diferencia de otros centros donde la rehabilitación pulmonar se hace durante la hospitalización, aumentando así los días de estancia hospitalaria. 
La mortalidad descrita por Rocco en Italia fue de $0,6 \%{ }^{13}$, aunque todos los casos estuvieron relacionados a progresión de la enfermedad con derrames neoplásicos. Abiuso reportó una mortalidad del $0,9 \%$, relacionada también con progresión de la enfermedad ${ }^{14}$. Esto contrasta con lo encontrado en el presente estudio, donde si bien se encontró una mortalidad superior al $6 \%$ en pacientes sometidos a VATS por monopuerto, estos decesos tampoco guardaron relación con el procedimiento quirúrgico y fueron consecuencias de la patología de base.

\section{Conclusiones}

La cirugía torácica asistida por video (VATS) por monopuerto se reconoce como una opción quirúrgica factible y segura para pleurectomía parietal y decorticación pulmonar. Este estudio refuerza la tendencia de mejores resultados postquirúrgicos, menores días de uso del tubo de tórax, uso de antibióticos, necesidad de UCI y días de estancia hospitalaria general con la técnica VATS por monopuerto comparado con la toracotomía abierta convencional.

\section{Cumplimiento de normas éticas}

Consentimiento informado: Este estudio es una revisión de historias clínicas retrospectiva, y como tal, no hay necesidad de un consentimiento informado. Se categorizó el estudio como investigación sin riesgo. El Comité de Ética Institucional aprobó el diseño y la metodología del estudio.

Conflictos de interés: Ninguno declarado por los autores.

Financiación: El desarrollo del trabajo fue financiado en su totalidad por los autores.

\section{Contribuciones de los autores:}

- Concepción y diseño del estudio: Anuar Alonso Sáez-Martínez, Daniel José Jaller-Salleg, Enrique Carlos Ramos-Clason, Paula Andrea Usta-Tirado.

- Adquisición de datos: Anuar Alonso Sáez-Martínez, Daniel José Jaller-Salleg, Enrique Carlos Ramos-Clason, Paula Andrea Usta-Tirado.

- Análisis e interpretación de datos: Anuar Alonso Sáez-Martínez, Daniel José Jaller-Salleg, Enrique Carlos Ramos-Clason, Paula Andrea Usta-Tirado.
- Redacción del manuscrito: Anuar Alonso Sáez-Martínez, Daniel José Jaller-Salleg, Enrique Carlos Ramos-Clason, Paula Andrea Usta-Tirado.

- Revisión crítica: Anuar Alonso Sáez-Martínez, Daniel José Jaller-Salleg, Enrique Carlos Ramos-Clason, Paula Andrea Usta-Tirado.

\section{Referencias}

1. Braimbridge M. Thoracoscopy: a historical perspective. Minimal access cardiothoracic surgery Philadelphia: WB Saunders. 2000.

2. Moreno-Sanz C, Almeida-Guevara A, Pascual-Pedreño A, Seoane-González J. Internet y la cirugía laparoscópica: un reto para el futuro. Cir Esp. 2003;73:178-82.

3. Cao C, Manganas C, Ang SC, Yan TD. A meta-analysis of unmatched and matched patients comparing video-assisted thoracoscopic lobectomy and conventional open lobectomy. Ann Cardiothorac Surg. 2012;1:16-23. https://doi.org/10.3978/j.issn.2225-319X.2012.04.18

4. Yan TD, Black D, Bannon PG, McCaughan BC. Systematic review and meta-analysis of randomized and nonrandomized trials on safety and efficacy of video-assisted thoracic surgery lobectomy for early-stage non-smallcell lung cancer. J Clin Oncol. 2009;27:2553-62. https://doi.org/10.1200/JC0.2008.18.2733

5. Lim E, Batchelor T, Shackcloth M, Dunning J, McGonigle N, Brush T, et al. Study protocol for VIdeo assisted thoracoscopic lobectomy versus conventional Open LobEcTomy for lung cancer, a UK multicentre randomised controlled trial with an internal pilot (the VIOLET study). BMJ Open. 2019;9:e029507.

https://doi.org/10.1136/ bmjopen-2019-029507

6. Ismail M, Nachira D, Meacci E, Ferretti GM, Swierzy $\mathrm{M}$, Englisch JP, et al. Uniportal video-assisted thoracic surgery in the treatment of pleural empyema. J Thorac Dis. 2018;10(Suppl 31):S3696-S3703.

https://doi.org/10.21037/jtd.2018.05.10

7. Van Middendorp LB, Franssen S, Gillissen S, Maessen JG, Hulsewé KWE, Vissers YLJ, et al. Uniportal video-assisted thoracoscopy is a safe approach in patients with empyema requiring surgery. J Thorac Dis. 2020;12:1460-6. https://doi.org/10.21037/jtd.2020.02.29

8. Abu Akar F, Gonzalez-Rivas D, Ismail M, Deeb M, Reichenshtein Y, Hadas-Halpern I, et al. Uniportal video-assisted thoracic surgery: the Middle East experience. J Thorac Dis. 2017;9:871-7. https://doi.org/10.21037/jtd.2016.11.89

9. Onaitis MW, Petersen RP, Balderson SS, Toloza E, Burfeind WR, Harpole DH, D'Amico TA. Thoracoscopic lobectomy is a safe and versatile procedure: experience with 500 consecutive patients. Ann Surg. 2006;244:420-5. https://doi.org/10.1097/01.sla.0000234892.79056.63 
10. Pan H, He J, Shen J, Jiang L, Liang W, He J. A meta-analysis of video-assisted thoracoscopic decortication versus open thoracotomy decortication for patients with empyema. J Thorac Dis. 2017;9:2006-14. https://doi.org/10.21037/ jtd.2017.06.109

11. Chan DTL, Sihoe ADL, Chan S, Tsang DST, Fang B, Lee TW, Cheng LC. Surgical treatment for empyema thoracis: is video-assisted thoracic surgery "better" than thoracotomy? Ann Thorac Surg. 2007;84:225-31. https://doi.org/10.1016/j.athoracsur.2007.03.019

12. Cardillo G, Carleo F, Carbone L, Di Martino M, Salvadori L, Petrella L, Martelli M. Chronic postpneumonic pleural empyema: comparative merits of thoracoscopic versus open decortication. Eur J Cardio-thoracic Surg. 2009;36:914-8.

https://doi.org/10.1016/j.ejcts.2009.06.017

13. Rocco G, La Rocca A, La Manna C, Scognamiglio F, D'Aiuto M, Jutley R, Martucci N. Uniportal video-assisted thoracoscopic surgery pericardial window. J Thorac Cardiovasc Surg. 2006;131:921-2.

14. Abiuso-Baesler VF, Lavanderos-Fernández J, Vega-Salas J, Fernández-Ruiz J, Salguero-Aparicio J, Cardemil-Herrera RG, Clavero-Ribes JM. Cirugía torácica videoasistida uniportal en dos centros universitarios. Experiencia inicial. Rev Cir. 2020;72:195-202. https://doi.org/10.35687/s2452-45492020003542 